\title{
Model-Based Correlation Measure for Nonuniformity Gain and Offset Parameters of Infrared Focal-Plane-Array Sensors`
}

\author{
César San Martin ${ }^{1,2}$ and Sergio N. Torres ${ }^{1}$ \\ ${ }^{1}$ Department of Electrical Engineering, University of Concepción. \\ Casilla 160-C, Concepción, Chile \\ cesanmartin@udec.cl, sertorre@udec.cl \\ ${ }^{2}$ Department of Electrical Engineering, University of La Frontera. \\ Casilla 54-D, Temuco, Chile \\ csmarti@ufro.cl
}

\begin{abstract}
In this paper, we proposed a model based correlation measure between gain and offset nonuniformity for infrared focal plane array (FPA) imaging systems. Actually, several nonuniformity correction methods perform correction of nonuniformities by means of gain and offset estimation in a detector-by-detector basis using several approach such as laboratory calibration methods, registration-based algorithm, and algebraic and statistical scene-based algorithm. Some statistical algorithms model the slow and random drift in time that the gain and offset present in many practical FPA applications by means of Gauss-Markov model, assuming that the gain and offset are uncorrelated. Due to this, in this work we present a study and model of such correlation by means of a generalized Gauss-Markov model. The gain and offset model-based correlation is validate using several infrared video sequences.
\end{abstract}

Keywords: Gauss-Markov Model, Image Sequence Processing, Infrared FPA, Signal Processing.

\section{Introduction}

Infrared (IR) cameras use an IR sensor to digitize the information, and due to its high performance, the most employed integrated technology in IR sensors is the Focal Plane Array (FPA). An IR-FPA is a die composed of a group of photodetectors placed in a focal plane forming a matrix of $X \times Y$ pixels, which gives the sensor the ability to collect the IR radiation.

Nonuniformity noise in IR imaging sensors, which is due to pixel-to-pixel variation in the detectors' responses, can considerably degrade the quality of IR

\footnotetext{
* This work was partially supported by Grants Milenio ICM P02-049, by FONDECYT 1060894 and DI-UFRO EP 120623. The authors wish to thank Ernest E. Armstrong (OptiMetrics Inc., USA) and Pierre Potet (CEDIP Infrared Systems, France) for collecting the data, and the United States Air Force Research Laboratory, Ohio, USA.
} 
images since it results in a fixed-pattern-noise (FPN) that is superimposed on the true image. Further, what makes matter worse is that the nonuniformity slowly varies over time, and depending on the FPA technology, this drift can take from minutes to hours. In order to solve this problem, several scene-based nonuniformity correction (NUC) techniques have been developed [1,2/3 4. Scenebased techniques perform the NUC using only the video sequences that are being imaged, not requiring any kind of laboratory calibration technique. Our group has been active in the development of novel scene-based algorithms for NUC based on statistical estimation theory. In [5]6] we have developed a Gauss-Markov model to capture the slow variation in the FPN and have utilized the model to adaptively estimate the nonuniformity in blocks of infrared video sequences using a Kalman Filter. In such work the gain and offset are assumed uncorrelated.

The principal contribution of this works is a study and model the detector gain and offset correlation. The model, called generalized Gauss-Markov model, is based in a measure of the correlation between gain and offset nonuniformity of infrared detectors. To study this correlation, we employ the detector parameters estimated from three published nonuniformity correction method: a neural network approach, a non-linear filtering method and statistical algorithm. With those method the correlation function is estimated and the Gauss-Markov model is then generalized. To validate the proposed model we tested with several infrared video sequences captured by an infrared camera.

This paper is organized as follows. In Sections 2 the IR-FPA model and the Gauss-Markov model are presented. In Section 3 the generalized Gauss-Markov model is developed. In Section 4 the correlation between gain and offset is measure and validate with sequence of real infrared data. In Section 5 the conclusions of the paper are summarized.

\section{Gauss-Markov Model for the Nonuniformity Detector Parameters}

In this paper, we model the pixel-to-pixel variation in the detectors' responses (nonuniformity) using the commonly used linear model for each pixel on the IR FPA. For the $(i j)^{\text {th }}$ detector, the measured readout signal $Y_{i j}(n)$ at a given time $n$ is represented by the approximate linear relation

$$
Y_{i j}(n)=X_{i j}^{(1)}(n) T_{i j}(n)+X_{i j}^{(2)}(n)+V_{i j}(n),
$$

where $X_{i j}^{(1)}(n)$ and $X_{i j}^{(2)}(n)$ are the gain and the offset of the $i j^{\text {th }}$ detector respectively and $T_{i j}(n)$ is the real incident infrared photon flux collected by the detector. The term $V_{i j}(n)$ represents the read-out noise associate with the $i j^{\text {th }}$ detector at time $n$. the subscript $i j$ is omitted with the understanding that all operations are performed on a pixel by pixel basis.

In practice, vectors of observations are captured over which the gain and offset are approximately constants. This is an acceptable assumption in applications where the observation vector is short. At a later time, other vectors of observation 
are captured for which these parameters may have drifted. This slow random variation of the gain and offset between consecutive vectors of observation is modeled by a Gauss-Markov process. It is also considered that the gain and offset at $k$ th vector-time are uncorrelated. The driver noise for the gain and offset are assumed gaussian individually and mutually uncorrelated among them. Mathematically, the Gauss-Markov state equation is given by

$$
\boldsymbol{X}_{k+1}=\boldsymbol{\Phi}_{k} \boldsymbol{X}_{k}+\boldsymbol{G}_{k} \boldsymbol{W}_{k}
$$

where $\boldsymbol{X}_{k+1}=\left[\begin{array}{c}X_{k}^{(1)} \\ X_{k}^{(2)}\end{array}\right]$ is the state vector at $k$ th vector time. $\boldsymbol{\Phi}=\left[\begin{array}{cc}\alpha_{k} & 0 \\ 0 & \beta_{k}\end{array}\right]$ is called the state transition matrix between the states at $k$ th and $k+1$ th time vector. $\alpha_{k}$ and $\beta_{k}$ represent the level of drift in the gain and offset between consecutive vectors of observation, respectively. The parameter $\alpha_{k}\left(\beta_{k}\right)$ is chosen according to the magnitude of the drift between the gain (offset) at state $k$ and the state $k+1 . G_{k}=\left[\begin{array}{ll}1 & 0 \\ 0 & 1\end{array}\right]$ is a identity matrix that randomly relates the driving noise vector $\boldsymbol{W}_{k}$ to the state vector $\boldsymbol{X}_{k} \cdot \boldsymbol{W}_{k}=\left[\begin{array}{c}W_{k}^{(1)} \\ W_{k}^{(2)}\end{array}\right], W_{k}^{(1)}$ and $W_{k}^{(2)}$ are the driving noise for the gain and offset process, respectively.

Finally, to complete the Gauss-Markov model, the observation model for the detector response is given by

$$
\boldsymbol{Y}_{k}=\boldsymbol{H}_{k} \boldsymbol{X}_{k}+\boldsymbol{V}_{k},
$$

where $\boldsymbol{Y}$ is the observation vector at $k$ th block, and the length of observation vector is $l_{k} \cdot \boldsymbol{H}_{k}=\left[\begin{array}{ccc}T(1) & 1 \\ \cdot & \cdot \\ \cdot & \cdot \\ \cdot & \cdot \\ T\left(l_{k}\right) & 1\end{array}\right]$ is the irradiance at $k$ th block. $\boldsymbol{V}_{k}$ is the vector of independent, additive temporal noise elements in the $k$ th block. The main assumption in the observation model is that the input irradiance $T(n)$ is a uniformly distributed random variable in the range $\left[T_{k}^{\min }, T_{k}^{\max }\right]$ constituting the range common to all detectors of possible irradiance levels prior to saturation. For a complete development for the gain and offset estimation of each detector in the FPA using an optimized recursion algorithm such that Kalman filter, see [5].

\section{Generalized Gauss-Markov Model}

In this paper, we propose generalize the model given at equation (1) by the next equation

$$
\boldsymbol{X}_{k+1}=\boldsymbol{\Phi}_{k} \boldsymbol{X}_{k}+\boldsymbol{G}_{k} \boldsymbol{W}_{k}
$$

where the transition matrix between the states at $k$ th and $k+1$ th time vector is modified to $\boldsymbol{\Phi}=\left[\begin{array}{cc}\alpha_{k} & \rho_{k} \\ \delta_{k} & \beta_{k}\end{array}\right]$ where $\rho_{k}$ and $\delta_{k}$ represent the cross correlation 
between the gain and offset in consecutive vectors of observation, respectively. The stability of the system of (3) is given by $\left|\lambda_{i}\left(\Phi_{k}\right)\right|<1$, i.e., the product of the eigenvalues must be lower than 1 . Note that if we multiplied (3) by $\boldsymbol{X}_{k+1-l}$ and applying the expectation value we obtain

$$
E\left[\boldsymbol{X}_{k+1} \boldsymbol{X}_{k+1-l}^{T}\right]=\boldsymbol{\Phi}_{k} E\left[\boldsymbol{X}_{k} \boldsymbol{X}_{k+1-l}^{T}\right]+G_{k} E\left[\boldsymbol{W}_{k} \boldsymbol{W}_{k-l}^{T}\right] G_{k}^{T},
$$

where $l=0,1, \ldots$ For a complete knowledge of the generalized Gauss-Markov model we need to determine the $\boldsymbol{G}_{k}$ and $\boldsymbol{\Phi}_{k}$ matrices. Now, in the next sections we present the development of the (4) on two cases: when $l=0$ and when $l>0$. The first case permits found the matrix $\boldsymbol{G}_{k}$ and the second case, permit found an expression for the transition matrix $\boldsymbol{\Phi}_{k}$.

\subsection{Solution for $G_{k}$ : Case Where $l=0$}

In this case, setting $l=0$, using (3) for $\boldsymbol{X}_{k+1}$, and replacing on (4) we obtain

$$
E\left[\boldsymbol{X}_{k+1} \boldsymbol{X}_{k+1}^{T}\right]=\boldsymbol{\Phi}_{k} E\left[\boldsymbol{X}_{k} \boldsymbol{X}_{k}^{T}\right] \boldsymbol{\Phi}_{k}^{T}+G_{k} E\left[\boldsymbol{W}_{k} \boldsymbol{W}_{k}^{T}\right] G_{k}^{T},
$$

and solve (5) for $E\left[\boldsymbol{W}_{k} \boldsymbol{W}_{k}^{T}\right]$ we obtain that

$$
E\left[\boldsymbol{W}_{k} \boldsymbol{W}_{k}^{T}\right]=G_{k}^{-1}\left\{E\left[\boldsymbol{X}_{k+1} \boldsymbol{X}_{k+1}^{T}\right]-\boldsymbol{\Phi}_{k} E\left[\boldsymbol{X}_{k} \boldsymbol{X}_{k}^{T}\right] \boldsymbol{\Phi}_{k}^{T}\right\} G_{k}^{-1^{T}},
$$

and assuming that $X_{k}$ is a stationary random variable

$$
E\left[\boldsymbol{X}_{k+1} \boldsymbol{X}_{k+1}^{T}\right]=E\left[\boldsymbol{X}_{k} \boldsymbol{X}_{k}^{T}\right]=\left[\begin{array}{cc}
\sigma_{a}^{2} & \sigma_{a b} \\
\sigma_{a b} & \sigma_{b}^{2}
\end{array}\right]
$$

and

$$
E\left[\boldsymbol{X}_{k+1} \boldsymbol{X}_{k+1}^{T}\right]-\boldsymbol{\Phi}_{k} E\left[\boldsymbol{X}_{k} \boldsymbol{X}_{k}^{T}\right] \boldsymbol{\Phi}_{k}^{T}=\left[\begin{array}{cc}
\sigma_{1}^{2} & \sigma_{0}^{2} \\
\sigma_{0}^{2} & \sigma_{2}^{2}
\end{array}\right]
$$

where $\sigma_{0}^{2}=(1-\alpha \beta-\delta \rho) \sigma_{a b}-\alpha \beta \sigma_{a}^{2}-\rho \beta \sigma_{b}^{2}, \sigma_{1}^{2}=\left(1-\alpha^{2}\right) \sigma_{a}^{2}-2 \alpha \rho \sigma_{a} b-\rho^{2} \sigma_{b}^{2}$ and $\sigma_{2}^{2}=\left(1-\beta^{2}\right) \sigma_{b}^{2}-2 \beta \delta \sigma_{a} b-\delta^{2} \sigma_{a}^{2}$, and considering $\boldsymbol{G}_{k}=\left[\begin{array}{cc}1 & g_{12} \\ g_{21} & 1\end{array}\right]$ we found that

$$
E\left[\boldsymbol{W}_{k} \boldsymbol{W}_{k}^{T}\right]=\left[\begin{array}{cc}
\sigma_{w a}^{2} & 0 \\
0 & \sigma_{w b}^{2}
\end{array}\right]
$$

i.e., $\boldsymbol{W}_{k}$ is uncorrelated only if $g_{12}$ and $g_{21}$ satisfies that

$$
g_{12}-\frac{g_{21} \sigma_{1}^{2}-\sigma_{0}^{2}}{g_{21} \sigma_{0}^{2}-\sigma_{2}^{2}}=0,
$$


with $\sigma_{w_{a}^{2}}^{2}=\sigma_{1}^{2}-2 g_{12} \sigma_{0}^{2}+g_{12} \sigma_{2}^{2}$ and $\sigma_{w_{b}}^{2}=\sigma_{2}^{2}-2 g_{21} \sigma_{0}^{2}+g_{21} \sigma_{1}^{2}$. For example, if $g_{21}=0$ then $g_{12}=\frac{\sigma_{0}^{2}}{\sigma_{2}^{2}}$ and

$$
\boldsymbol{G}_{k}=\left[\begin{array}{cc}
1 & \frac{\sigma_{0}^{2}}{\sigma_{2}^{2}} \\
0 & 1
\end{array}\right]
$$

which implies that the $\boldsymbol{W}_{k}$ is uncorrelated. Similarly, the expectation of $\boldsymbol{W}_{k}$ can be obtained from (3) by means

$$
E\left[\boldsymbol{W}_{k}\right]=\boldsymbol{G}_{k}^{-1}\left[I-\boldsymbol{\Phi}_{k}\right] E\left[\boldsymbol{X}_{k}\right]=\boldsymbol{G}_{k}^{-1}\left[I-\boldsymbol{\Phi}_{k}\right] E\left[\boldsymbol{X}_{0}\right]
$$

\subsection{Solution for $\Phi_{k}$ : Case Where $l>0$}

In this case, replacing $l>0$ on (4) we obtain

$$
E\left[\boldsymbol{X}_{k+1} \boldsymbol{X}_{k+1-l}^{T}\right]=\boldsymbol{\Phi}_{k} E\left[\boldsymbol{X}_{k} \boldsymbol{X}_{k+1-l}^{T}\right],
$$

and expanding (8) on each elements of $\boldsymbol{X}$, using correlation function notation and solve for $\boldsymbol{\Phi}_{k}$ we obtain

$$
\boldsymbol{\Phi}_{k}=\left[\begin{array}{ll}
R_{X^{(1)} X^{(1)}}(l) & R_{X^{(1)} X^{(2)}}(l) \\
R_{X^{(2)} X^{(1)}}(l) & R_{X^{(2)} X^{(2)}}(l)
\end{array}\right]\left[\begin{array}{ll}
R_{X^{(1)} X^{(1)}}(l-1) & R_{X^{(1)} X^{(2)}}(l-1) \\
R_{X^{(2)} X^{(1)}}(l-1) & R_{X^{(2)} X^{(2)}}(l-1)
\end{array}\right]^{-1},
$$

or

$$
\boldsymbol{\Phi}_{k}=\boldsymbol{R}_{\boldsymbol{X}}(l) \boldsymbol{R}_{\boldsymbol{X}}^{-1}(l-1) .
$$

Note that in [5] they assume that gain and offset are uncorrelated, $\alpha_{0}=\alpha_{1}=$ $\ldots=\alpha_{k}=\alpha$ and $\beta_{0}=\beta_{1}=\ldots=\beta_{k}=\beta$. Replacing this assumption on (9) results

$$
\boldsymbol{\Phi}_{k}=\left[\begin{array}{cc}
\frac{R_{X^{(1)} X^{(1)}}(l)}{R_{X^{(1)} X^{(1)}(l-1)}} & 0 \\
0 & \frac{R_{X(2)}{ }^{(2)}(l)}{R_{X^{(2)} X^{(2)}}(l-1)}
\end{array}\right]=\left[\begin{array}{ll}
\alpha & 0 \\
0 & \beta
\end{array}\right],
$$

this mean that the gain and offset are signals with exponential correlation function, i.e., following a Gauss-Markov model given in (11). Note that (11) correspond a particular case of (9), called the generalized transition matrix. In the next section we present the methodology to obtain an estimation of $\boldsymbol{\Phi}$ using (10), i.e., based on estimate the correlation functions $\boldsymbol{R}_{\boldsymbol{X}}(l)$ and $\boldsymbol{R}_{\boldsymbol{X}}(l-1)$ obtained using some NUC published method.

\section{Estimation and Validation of the Generalized Model}

In this section we obtain an estimation of $\boldsymbol{\Phi}_{k}$ from (10) using three NUC published method: the neural networks approach $1 / 234$, nonlinear filtering 78 
and statistical algorithm [9]. Briefly, the neural networks approach use a hidden layer like a two-point NUC neurons that models the gain and offset of each detectors. This NUC technique is derived using and adaptive least-square (LMS) approach, making it a comparison between neighboring pixels as new frames of data are input (the retina-like processing). The second NUC method, nonlinear filtering, is used to obtain an individual estimation of gain and offset with recursive least-square (RLS) based on minimizing the error between a preliminary true scene estimation and the corresponding frame corrected for each pixel. This preliminary estimation of true irradiance is performed by a spatial nonuniformity filtering of readout data reducing the spatial signal resolution that is restored by the RLS algorithm. Both NUC methods, LMS and RLS based, perform the detector parameters estimation in a frame by frame basis. On the other hand, the statistical algorithm assumes that the irradiance at each detector is a uniformly distributed random variable and that the gain and offset are temporally constant within certain blocks of time. This mean that the gain and offset estimation is performed in a block of frame basis, i.e., when a new block of raw data arrives, the method perform the NU correction by a finite-impulse response filter and then, the temporal filter is adapted to the changes in the detector parameters and the gain and offset are updated to the next arrivals of raw data.

Now, to obtain an estimation for the gain and offset $\left(X^{(1)}, X^{(2)}\right)$ we use real infrared data captured by the $128 \times 128$ InSb FPA cooled camera (Amber Model AE-4128) operating in $3-5 \mu \mathrm{m}$ range. The sequences were collected at $1 \mathrm{PM}$ with 3000 frames collected at a rate of 30 frames/s and each pixel was quantized in 16 bit integers. Using this raw data, we able to estimate for each method the detector parameters correlation function $\boldsymbol{R}_{\boldsymbol{X}}(1)$ and $\boldsymbol{R}_{\boldsymbol{X}}(0)$ to obtain a suitable estimation of $\boldsymbol{\Phi}_{k}$. Additionally, in this work we assume that $\boldsymbol{\Phi}_{0}=\boldsymbol{\Phi}_{1}=\ldots=$ $\boldsymbol{\Phi}_{k}=\boldsymbol{\Phi}$. Now, the correlation function between $X^{i}$ and $X^{j}$ with $i=1,2$ and $j=1,2$, can be estimated by 10 .

$$
\hat{R}_{X^{(i)} X^{(j)}}^{n}\left(l^{\prime}\right)=\frac{1}{n} \sum_{k=0}^{n-1} X^{(i)}(k) X^{(j)}\left(k+l^{\prime}\right)
$$

where $\hat{R}_{X^{(i)} X^{(j)}}^{n}\left(l^{\prime}\right)$ is the estimation for $R_{X^{(i)} X^{(j)}}(1)$ and $R_{X^{(i)} X^{(j)}}(0)$ when $i=1,2, j=1,2$ and $l^{\prime}=0,1$. In (12) the knowledge of $X^{(1)}$ and $X^{(2)}$ at each time $k$ is required. Since from the three NUC method we have an estimation of the gain and offset we can express (12) by the following recursive algorithm

$$
\hat{R}_{X^{(i)} X^{(j)}}^{n}\left(l^{\prime}\right)=\frac{n-1}{n} \hat{R}_{X^{(i)} X^{(j)}}^{n-1}\left(l^{\prime}\right)+\frac{1}{n} X^{(i)}(n-1) X^{(j)}\left(n-1+l^{\prime}\right),
$$

and then, using (9) we finally obtain the estimated $\boldsymbol{\Phi}$ for each method.

In summary, to obtain an estimation for $\boldsymbol{\Phi}$ we needed to make the following steps: i) using real infrared sequence captured by Amber camera, estimate the gain and offset by three NUC methods, ii) estimate the correlation function for $\boldsymbol{R}_{\boldsymbol{X}}(1)$ and $\boldsymbol{R}_{\boldsymbol{X}}(0)$ using (13) and finally, iii) obtain the estimated $\boldsymbol{\Phi}$ for each method using (10). 
To validate the parameters of each model, we considered two aspect: the NUC performance and the estimation error. For study the NUC capability of each model we use the roughness parameters defined by

$$
\rho(f)=\frac{\|h * f\|_{1}+\left\|h^{T} * f\right\|_{1}}{\|f\|_{1}},
$$

where $h$ is a horizontal mask, $\|f\|_{1}$ is the $L_{1}$ norm of frame $f$ and $*$ represents discrete convolution. In the other hand, to validate the model we use equation (2) in order to calculate the mean-square error (MSE) between the raw data $\boldsymbol{Y}_{k}$ and the estimation $\hat{\boldsymbol{Y}}_{k}\left(\boldsymbol{\Phi}^{j}\right)$ for each model, given by [1]

$$
M S E=\frac{1}{N} \sum_{k=0}^{N-1}\left[\boldsymbol{Y}_{k}-\hat{\boldsymbol{Y}}_{k}\left(\boldsymbol{\Phi}^{j}\right)\right]^{T}\left[\boldsymbol{Y}_{k}-\hat{\boldsymbol{Y}}_{k}\left(\boldsymbol{\Phi}^{j}\right)\right],
$$

where $\hat{\boldsymbol{Y}}_{k}\left(\boldsymbol{\Phi}^{j}\right)=\boldsymbol{H} \boldsymbol{X}_{k}=\boldsymbol{H} \boldsymbol{\Phi} \boldsymbol{X}_{k-1}$ is the a priori estimate of $\hat{\boldsymbol{Y}}_{k}$ based on the model $\boldsymbol{\Phi}^{j}$ for $j=1,2,3$.

Table 1. Result on Validation for different models

\begin{tabular}{cccc}
\hline Model & $\left|\lambda_{i}\left(\phi^{j}\right)\right|$ & $\rho(f)$ & MSE \\
\hline $\boldsymbol{\Phi}^{1}$ & $7.59 \times 10^{-9}$ & 2.2425 & 0.8844 \\
$\boldsymbol{\Phi}^{2}$ & 0.2353 & 2.2127 & 0.8591 \\
$\boldsymbol{\Phi}^{3}$ & 0.0244 & 2.1313 & 0.1909 \\
\hline
\end{tabular}

Following the previous procedure we obtain three generalized models corresponding to each NUC algorithm given by:

$$
\begin{aligned}
& \boldsymbol{\Phi}^{1}=\left[\begin{array}{cc}
0.5026 & -0.5026 \\
-0.5026 & 0.5026
\end{array}\right] \\
& \boldsymbol{\Phi}^{2}=\left[\begin{array}{cc}
0.8834 & -0.7384 \\
-0.7384 & 0.8835
\end{array}\right] \\
& \boldsymbol{\Phi}^{3}=\left[\begin{array}{cc}
0.6031 & -0.5505 \\
-0.1252 & 0.1548
\end{array}\right]
\end{aligned}
$$

where $\boldsymbol{\Phi}^{1}, \boldsymbol{\Phi}^{2}$ and $\boldsymbol{\Phi}^{3}$ correspond to the model obtained with neural network approach, nonlinear filtering and statistical algorithm respectively. Note that all NUC methods presented are stable (see Table 1) and shows that $X^{(1)}$ and $X^{(2)}$ are correlated. This mean that the assumption that the gain and offset in consecutive vector of observation are uncorrelated is not valid. Even more, whereas LMS and RLS algorithm exhibit a large correlation, the statistical algorithm shows that the gain strongly is influenced by the gain and offset of the previous state, i.e., we can assume that the offset indirectly is estimate from gain. It is very important conclusion because we need the knowledge only of one state to reduce NU. 


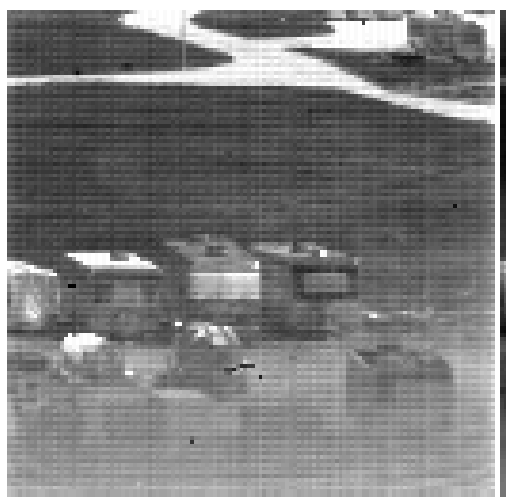

(a)

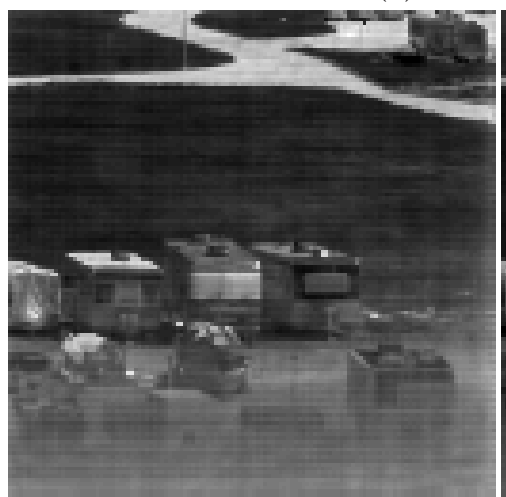

(c)

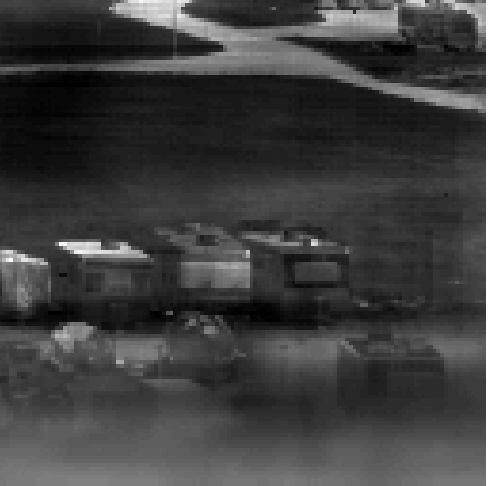

(b)

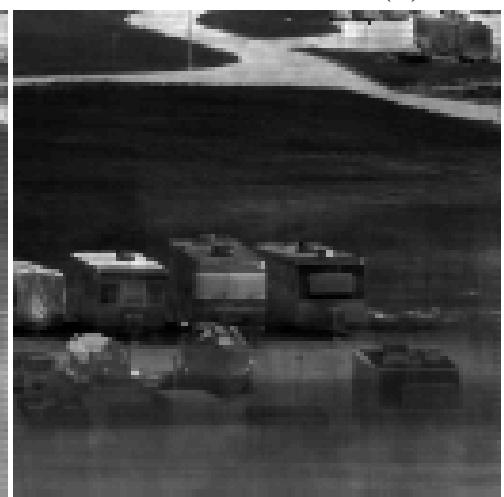

(d)

Fig. 1. The 2220 - th frame form 1 PM sequence captured with Amber infrared camera: a) the corrupted raw frame, corrected frame with b) neural network approach, c) nonlinear filtering, and d) statistical algorithm. It can be seen by using only the naked eyes that a good NUC is obtained from the three NUC methods.

In Table 1 , the $\left|\lambda_{i}\left(\Phi^{j}\right)\right|$ for each model, spatial average of $\rho$ and MSE parameters are presented. We can observe that the best performance is obtained for statistical algorithm $\left(\boldsymbol{\Phi}^{3}\right)$. Additionally, from equations (16), (17) and (18) we can observe that as the magnitude of the drift between the gain (offset) at state $k$ and state $k+1$ is more different (i.e., the correlation between gain and offset is considered), the NU correction and the model estimation are improved. This in addition confirms the assumption of the gain and offset are temporally constant in a block of time. Finally, for illustration proposed, the LMS-based, RLS-based and statistical algorithm were applied to the 2220-frame image sequence, and the results is shown in Figure 1. In this figure, Fig. 1(a) correspond to the corrupted frames, Fig. 1(b), Fig. 1(c) and Fig. 1(d) shows the corrected frames with neural network approach, nonlinear filtering, and statistical algorithm respectively. This figure clearly shows that the statistical method present a good reduction of FPN. 


\section{Conclusions}

In this paper a study of correlation between gain and offset is present. It was shown experimentally using real IR data that the assumption of gain and offset is uncorrelated is not valid on some NUC scene-based method. Indeed, when the asymmetry is more notable in the generalized transition matrix, i.e., the magnitude of the drift between the gain (offset) at consecutive block of frames is more different, the method has shown an improved in reduction of nonuniformity and in estimation error. This allows to conclude that the gain and offset are temporally constant in a block of time and the gain and offset are correlated. From this assumption, we can reformulate the model present on [5]. Future work consider a generalized Gauss-Markov model by obtain an adaptive estimation of nonuniformity using a Kalman filter and an evaluation to compare with uncorrelated Gauss-Markov model.

\section{References}

1. Scribner, D., Sarkady, K., Kruer, M.: Adaptive Nonuniformity Correction for Infrared Focal Plane Arrays using Neural Networks. Proceeding of SPIE. 1541, 100109 (1991)

2. Scribner, D., Sarkady, K., Kruer, M.: Adaptive Retina-like Preprocessing for Imaging Detector Arrays. Proceeding of the IEEE International Conference on Neural Networks. 3, 1955-1960 (1993)

3. Torres, S., Vera, E., Reeves, R., Sobarzo, S.: Adaptive Scene-Based Nonuniformity Correction Method for Infrared Focal Plane Arrays. Proceeding of SPIE. 5076, 130-139 (2003)

4. Vera, E., Torres, S.: Fast Adaptive Nonuniformity Correction for Infrared Focal Plane Arrays. Journal on Applied Signal Processing EURASIP (2005)

5. Torres, S., Hayat, M.: Kalman Filtering for Adaptive Nonuniformity Correction in Infrared Focal Plane Arrays. The JOSA-A Opt. Soc. of America. 20, 470-480 (2003)

6. Torres, S., Pezoa, J., Hayat, M.: Scene-based Nonuniformity Correction for Focal Plane Arrays Using the Method of the Inverse Covariance Form. OSA App. Opt. Inf. Proc. 42, 5872-5881 (2003)

7. Torres, F., Torres, S., Martn, C.S.: A Recursive Least Square Adaptive Filter for Nonuniformity Correction of Infrared Image Sequences. In: Sanfeliu, A., Cortés, M.L. (eds.) CIARP 2005. LNCS, vol. 3773, pp. 540-546. Springer, Heidelberg (2005)

8. Torres, F., Martin, C.S., Torres, S.: A RLS Filter for Nonuniformity and Ghosting Correction of Infrared Image Sequences. Sequences. In: Martínez-Trinidad, J.F., Carrasco Ochoa, J.A., Kittler, J. (eds.) CIARP 2006. LNCS, vol. 4225, pp. 464473. Springer, Heidelberg (2006)

9. Hayat, M., Torres, S., Amstrong, E., Cain, S., Yasuda, B.: Statistical Algorithm fo Nonuniformity Correction in Focal Plane Arrays. Applied Optics 38, 773-780 (1999)

10. Therrien, C.W.: Discrete Random Signal and Statistical Signal Processing. Prentice Hall Press, USA (1992)

11. Pezoa, J., Hayat, M., Torres, S., Rahman, M.: Multimodel Kalman Filtering for Adaptive Nonuniformity Correction in Infrared Sensors. JOSA A 23, 1282-1291 (2006) 